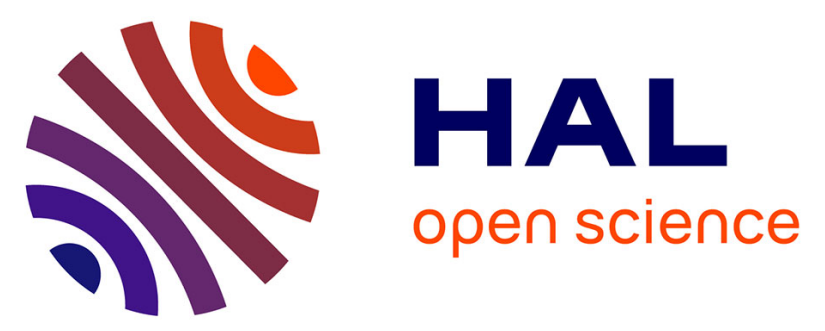

\title{
Effects of Cauchy integral formula discretization on the precision of IF estimation; unified approach to complex-lag distribution and its counterpart $\mathrm{L}$ form
} Irena Orovic, Srdjan Stankovic, Cornel Ioana

\section{- To cite this version:}

Irena Orovic, Srdjan Stankovic, Cornel Ioana. Effects of Cauchy integral formula discretization on the precision of IF estimation; unified approach to complex-lag distribution and its counterpart L form. IEEE Signal Processing Letters, 2009, 16 (4), pp.327-330. 10.1109/LSP.2008.2011711 · hal-00349537

\section{HAL Id: hal-00349537 \\ https://hal.science/hal-00349537}

Submitted on 31 Dec 2008

HAL is a multi-disciplinary open access archive for the deposit and dissemination of scientific research documents, whether they are published or not. The documents may come from teaching and research institutions in France or abroad, or from public or private research centers.
L'archive ouverte pluridisciplinaire $\mathbf{H A L}$, est destinée au dépôt et à la diffusion de documents scientifiques de niveau recherche, publiés ou non, émanant des établissements d'enseignement et de recherche français ou étrangers, des laboratoires publics ou privés. 


\title{
Effects of Cauchy integral formula discretization on the precision of IF estimation; unified approach to complex-lag distribution and its counterpart $\mathrm{L}$ form
}

\author{
Irena Orovic, Srdjan Stankovic, Cornel Ioana
}

\begin{abstract}
An interpretation of time-frequency (TF) distribution concentration based on discretization of Cauchy's integral formula, is provided. In order to increase the accuracy of instantaneous frequency (IF) estimation, two solutions are considered: increasing the number of points for integration and multiple integrations using the same number of points (it corresponds to $L$ form of $T F$ distribution). In practical applications, $\mathrm{L}$ form of the fourth order complex-lag distribution has shown excellent results. For this case the analysis of noise influence is provided, as well.
\end{abstract}

Index Terms - time-frequency distributions, complex analysis

\section{INTRODUCTION}

$\mathrm{T}$ HE concentration is one of the most important properties of the TF distributions. In order to obtain high concentration in the TF plane, various TF distributions have been derived [1]-[9]. Concentration improvement is especially important for the instantaneous frequency estimation of highly non-stationary signals [3]-[8].

In this letter we will present an approach which can produce completely concentrated representation along the IF. It is based on the concept of Cauchy's integral formula discretization. Some interesting and specific solutions for concentration improvement are analyzed. It will be shown that the efficiency of IF estimation can be improved either by increasing the number of discretization points on the circle or by performing multiple integrations over the circle. The first solution leads to the definition of the novel general forms of TF distributions, whose special cases correspond to various existing TF distributions [6]-[8]. The second one introduces their $L$ forms. Here, it is important that multiple integrations approach does not influence significantly the realization complexity, since it can be obtained recursively from the basic distribution form like in [6].

\section{INTERPRETING AND IMPROVING TF DISTRUBUTION CONCENTRATION}

\section{A. IF Calculation based on the Cauchy's integral formula}

Manuscript received August 10, 2008. Authors are with the Electrical Engineering Department, University of Montenegro, 81000 Podgorica, Montenegro. Corresponding author: e-mails: ()@ cg.ac.yu.
According to the Cauchy's integral formula from the complex analysis, a holomorphic function $f(z)$ defined on the closed disc $D=\left\{z:\left|z-z_{0}\right| £ r\right\}$ can be completely determined by its values on the boundary circle $C$ of the disc:

$$
f(t)=\frac{1}{2 p j} \tilde{\mathbb{\mathbf { N }}} \frac{f(z)}{z-t} d z .
$$

Consequently, the derivatives of the function $f$ are obtained using the integration over $C$ as follows:

$$
f^{K}(t)=\frac{K !}{2 p j} \tilde{\mathrm{d}}_{C} \frac{f(z)}{(z-t)^{K+1}} d z
$$

Let consider a signal in the form $s(t)=r x^{j f(t)}$, with constant amplitude $r$ and a phase function $f(t)$. In order to provide an estimation of the IF, we may assume that circle $C$ is centered at instant $t$ and $z=t+t e^{j q}$, where $\tau$ is the radius of the circle [8]. Thus, the first phase derivative can be obtained as:

$f^{\prime}(t)=\frac{1}{2 p j} \tilde{\mathbb{N}}_{C} \frac{f\left(t+t e^{j q}\right)}{\left(t e^{j q}\right)^{2}} d\left(t e^{j q}\right)=\frac{1}{2 p t}{ }_{0}^{2 p} f\left(t+t e^{j q}\right) e^{-j q} d q$.

Reducing the circle radius $\tau$ by some scaling factor $L$ results in $L$ (multiple) integrations as follows:

$$
f^{\prime}(t) t=\frac{L}{2 p}{ }_{0}^{2 p} f\left(t+\frac{t}{L} e^{j q}\right) e^{-j q} d q .
$$

In the $\mathrm{TF}$ analysis, $\tau$ has been introduced into the definition of the TF distributions as a lag coordinate. Thus, scaling of the lag coordinate by $L$ provides increasing of accuracy upon the IF estimation. The discretization of (3), leads to the suitable form for further analysis [8]:

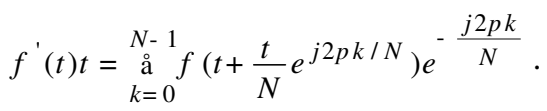

Note that, in order to obtain the IF that is concentrated along $f^{\prime}(t)$, the lag coordinate $t$ is divided by the number of discretization points $N$. For large $N$, almost an ideally concentrated IF could be obtained. The right side of (5) represents the phase of the complex-lag signal's moment [8]: 


$$
M(t, t)={\underset{\tilde{O}}{k=0}}_{k=1}^{N} s^{-j 2 p k / N}\left(t+\frac{t}{N} e^{j 2 p k / N}\right) .
$$

The corresponding TF distributions are obtained by applying the Fourier transform to the moment function.

\section{B. Analysis of IF estimation precision}

Consider the general case of two points on the circle: $\frac{t}{2(a+j b)}$ and $\frac{t}{2(-a-j b)}$, located symmetrically around instant $t$, Fig 1.a. In this case the moment function can be written as:

$$
M(t, t)=s^{(a+j b)}\left(t+\frac{t}{2(a+j b)}\right) s^{(-a-j b)}\left(t+\frac{t}{2(-a-j b)}\right) .
$$

Applying the Taylor series expansion to the phase of $M(t, t)$ will produce:

$$
\phi_{M}(t, \tau)=\phi^{\prime}(t) \tau+\phi^{(3)}(t) \frac{\tau^{3}}{2^{2} 3 !(a+j b)^{2}}+\phi^{(5)}(t) \frac{\tau^{5}}{2^{4} 5 !(a+j b)^{4}}+\ldots
$$

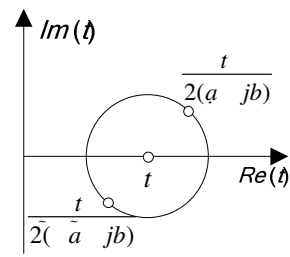

a)

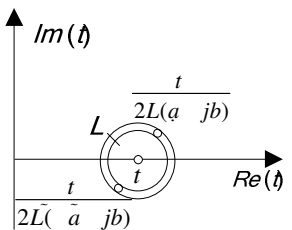

d)

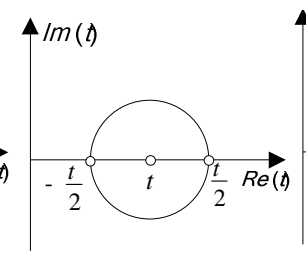

b)

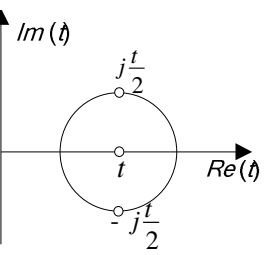

c)

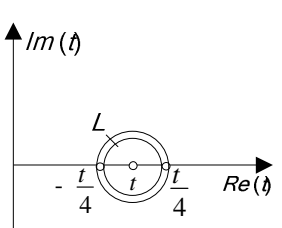

e)

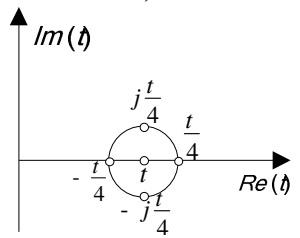

f)
Fig 1. Illustration of circle points around instant $t:$ a) two points- general case, b) points on real axes, c) points on imaginary axes, d) two points $L$ times, e) two points on real axes $L$-times, f) four points

Observe that all terms apart from $f^{\prime}(t) \times$ (terms containing the third and higher order odd derivatives), represent the integration error. This error is reflected as a spread factor that affects the concentration. The TF representation based on the moment function (7) is defined as:

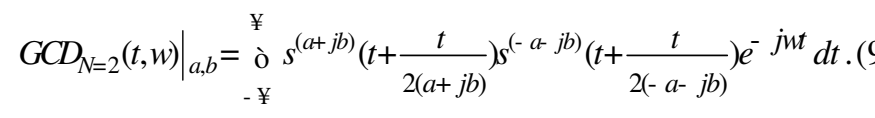

It provides ideal concentration for linear IF. However, note that in general case, some terms in (8) may even result in amplitude modulations. They could be avoided for the purpose of the IF estimation, as it will be discussed later. In the sequel, we will analyze few interesting cases.

Case 1): $(\mathrm{a}, \mathrm{b})=(1,0)$. The real valued points $\frac{t}{2}$ and $-\frac{t}{2}$ are considered (Fig 1.b). In this case, (9) corresponds to the wellknown Wigner distribution. Note that power -1 is used instead of conjugate and the marginal properties are not preserved, but it has the same influence on the IF estimation. The spread factor for the Wigner distribution is given in Table I.
Case 2): $(a, b)=(0,1)$. Regarding the IF estimation, almost the same results as in the previous case are obtained if the points $j \frac{t}{2}$ and $-j \frac{t}{2}$ (Fig 1.c) are used. The corresponding signal moment is: $M(t, t)=s^{-j}\left(t+j \frac{t}{2}\right) s^{j}\left(t-j \frac{t}{2}\right)$, while its phase contains the same derivatives as in the Wigner distribution.

Case 3): An interesting case which eliminates the $3^{\text {rd }}, 7^{\text {th }}$, $11^{\text {th }}$, and higher order derivatives can be obtained for $a=b=\sqrt{2} / 2$. In this case the Taylor series expansion of the moment phase function is obtained in the form: $\phi_{M}(t, \tau)=\dot{\phi}^{\prime}(t) \tau-j \phi^{(3)}(t) \frac{\tau^{3}}{2^{2} 3 !}-\phi^{(5)}(t) \frac{\tau^{5}}{2^{4} 5 !}+\ldots \cdot \quad$ Apparently, the derivatives of order $4 n-1 \quad(n=1,2, \ldots)$ yield to amplitude modulation terms. Thus, to avoid these terms for the purpose of the IF estimation, a slight modification of $M(t, t)$ should be used: $e^{j \text { xangle }(M(t, t))}$.

Note that these forms of distributions (Cases 2 and 3) are not energetic, because the unit amplitude is considered.

For non-linear IF, the spread factor might be significant and the inner interferences appear. Therefore, in order to improve concentration for non-linear signal phase, the more accurate integration should be considered. It can be realized as the integration over $L$ circles with $L$ times smaller radius, as it is given by (4). An illustration for two overall points is shown in Fig 1.d. Consequently, the $L$ form of the distribution defined by (9) results:

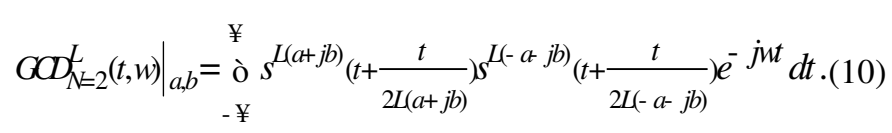

For instance, in the case $L=2$, the precision of IF estimation is improved by using twice the real axes points $\frac{t}{4}$ and $-\frac{t}{4}$ (Fig 1.e). Thereby the $L$-Wigner distribution is obtained as [6]:

$$
G C D_{N=2}^{L=2}(t, w)=\underset{-}{\stackrel{\ddagger}{\ddagger}} s^{2}\left(t+\frac{t}{4}\right) s^{-2}\left(t-\frac{t}{4}\right) e^{-j w t} d t .
$$

The $L$-Wigner distribution reduces the spread factor better than the Wigner distribution (Table I), providing higher distribution concentration.

The precision of integration (i.e. IF estimation) can be further improved by using higher number of different points instead of using multiple integrations through the same points. For two pairs of symmetrical points $\left[ \pm \frac{t}{4\left(a_{i}+j b_{i}\right)}\right], i=1,2$, the moment phase expansion is given by: $\phi_{M}(t, \tau)=\phi^{\prime}(t) \tau+$

$$
+2 \phi^{(3)}(t) \frac{\tau^{3}}{4^{3} 3 !}\left(\frac{1}{\left(a_{1}+j b_{1}\right)^{2}}+\frac{1}{\left(a_{2}+j b_{2}\right)^{2}}\right)+2 \phi^{(5)}(t) \frac{\tau^{5}}{4^{5} 5 !}\left(\frac{1}{\left(a_{1}+j b_{1}\right)^{4}}+\frac{1}{\left(a_{2}+j b_{2}\right)^{4}}\right)+\ldots .
$$

By suitable selection of $a_{1}, b_{1}, a_{2}$, and $b_{2}$, some odd derivatives may also disappear or significantly be reduced. For example, it is easy to see that for $a_{1}=-a_{2}=b_{1}=b_{2}$ all the derivatives of order $4 n-1, n=1,2, \ldots$, will be annulled.

The corresponding TF distribution is defined as: 


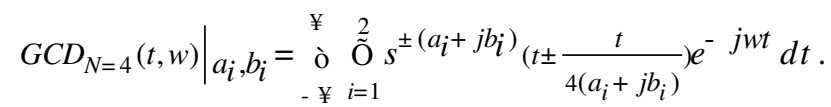

Observe that in the case $\left(a_{1}, b_{1}, a_{2}, b_{2}\right)=(1,0,0,1)$ (Fig 1.d), a form of known complex-lag distribution $(N=4)$ is obtained [7]:

$$
G C D_{N=4}(t, w)=\underset{-}{\stackrel{\ddagger}{o}} s\left(t+\frac{t}{4}\right) s^{-1}\left(t-\frac{t}{4}\right) s^{-j}\left(t+j \frac{t}{4}\right) s^{j}\left(t-j \frac{t}{4}\right) e^{-j w t} d t .
$$

Its spread factor is given in Table $\mathrm{I}\left(3^{\text {rd }}\right.$ row $)$. In addition, it is interesting to observe: $\left(a_{1}, b_{1}, a_{2}, \mathrm{~b}_{2}\right)=(1 / 2, \sqrt{3} / 2, \sqrt{3} / 2,1 / 2)$. By using the previous modification $e^{j \times a n g l e(M(t, t))}$, we will obtain the spread factor of (13) divided by 2 . Also, an advantage of using the points with smaller imaginary part is in reducing the miscalculations that may appear in the realization of signal with complex lag argument [7].

For signals with higher non-stationarity, the precision can be additionally improved by using the concept of multiple integrations. Thus, similarly as in (10), the $L$ form of (12) can be used. For instance, the $L$ form of $\operatorname{GCD}_{N=4}(t, w)$ is defined as:

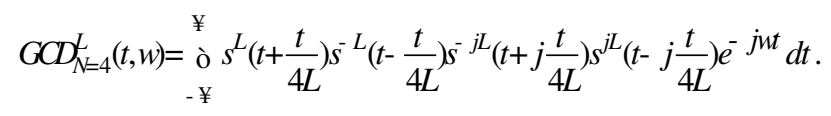

TABLE I. SPREAD FACTORS FOR SOME TF DISTRIBUTIONS

\begin{tabular}{|c|c|}
\hline Distribution & Spread factor \\
\hline $\begin{array}{c}\text { Wigner distribution, } \\
\left(G C D_{N}, N=2\right)\end{array}$ & $Q(t, \tau)=\phi^{(3)}(t) \frac{\tau^{3}}{2^{2} 3 !}+\phi^{(5)}(t) \frac{\tau^{5}}{2^{4} 5 !}+\ldots$ \\
\hline $\begin{array}{c}\text { L-Wigner distribution } \\
G C D_{N=2}^{L}\end{array}$ & $Q(t, \tau)=\phi^{(3)}(t) \frac{\tau^{3}}{2^{2} 3 ! L^{2}}+\phi^{(5)}(t) \frac{\tau^{5}}{2^{4} 5 ! L^{4}}+\ldots$ \\
\hline$G C D_{N=4}$ & $Q(t, \tau)=\phi^{(5)}(t) \frac{\tau^{5}}{4^{4} 5 !}+\phi^{(9)}(t) \frac{\tau^{9}}{4^{8} 9 !}+\ldots$ \\
\hline$G C D_{N=4}^{L=2}$ & $Q(t, \tau)=\phi^{(5)}(t) \frac{\tau^{5}}{4^{6} 5 !}+\phi^{(9)}(t) \frac{\tau^{9}}{4^{12} 9 !}+\ldots$ \\
\hline$G C D_{N=s}^{L}$ & $Q(t, \tau)=\phi^{s+1}(t) \frac{\tau^{s+1}}{s^{s}(s+1) ! L^{s}}+\phi^{2 s+1}(t) \frac{\tau^{2 s+1}}{s^{2 s}(2 s+1) ! L^{2 s}}+\ldots$ \\
\hline
\end{tabular}

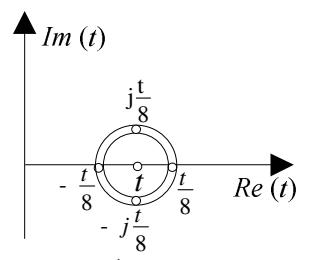

a)

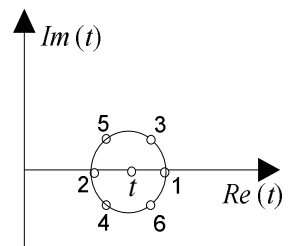

b)

$[1,2,3,4,5,6=]\left[\frac{t}{6},-\frac{t}{6},\left(\frac{\sqrt{3}+j}{2}\right) \frac{t}{6},\left(\frac{-\sqrt{3}-j}{2}\right) \frac{t}{6},\left(\frac{-\sqrt{3}+j}{2}\right) \frac{t}{6},\left(\frac{\sqrt{3}-j}{2}\right) \frac{t}{6}\right]$

Fig 2: Illustration of circle points around instant $t$ for: a) circle $G C D_{N=4}^{L=2}(t, w)$, b) $G C D_{N=6}(t, w)$

The integration points for $L=2$ are illustrated in Fig 2.a. The terms within the spread factor for $G C D_{N=4}^{L=2}(t, w)$ are reduced comparing to $G C D_{N=4}(t, w)$ ( Table I). Regarding the complexity of realization the $L$ form of complex-lag distribution (for even
$L)$ can be easily obtained by performing the recursive realization as follows:

$$
G C D_{N=4}^{L}(t, w)=\frac{1^{-}}{p} \underset{\ddagger}{\grave{o}} G C D_{N=4}^{L / 2}(t, w+q) G C D_{N=4}^{L / 2}(t, w-q) d q \cdot
$$

This form is very suitable for practical applications and will be further analyzed in the next section.

By considering more points on the circle, an additional improvement of IF estimation accuracy can be provided. However, the realization complexity increases. An illustration of some points on the circle for $N=6$ is given in Fig 2.b. Ideally, for an arbitrarily high number of points $N$ and integrations $L$, the spread factor tends to zero. The corresponding $L$ form of the $N$-th order complex-lag distribution can be defined as follows:

$G C D_{N}^{L}(t, w)=\stackrel{\substack{\text { o } \\-\ngtr}}{N / 2-1} \tilde{O}_{i=1}^{ \pm L\left(a_{i}+j b_{i}\right)}\left(t \pm \frac{t}{N \rtimes\left(a_{i}+j b_{i}\right)}\right) e^{-j w t} d t .(16$

An interesting case is obtained for equidistant points: $\left\{ \pm\left(a_{i}+j b_{i}\right) \mid i=1, \ldots, N / 2-1\right\}=\left\{e^{j 2 p k / N} \mid k=1, \ldots, N-1\right\}$. Note that for $L=1$, it corresponds to the generalized complex-lag $\mathrm{TF}$ distribution [8]. It can be seen from Table I (last row) that the distribution spread factor can be arbitrarily reduced by the suitable selection of parameters $L$ and $N$ (distribution order).

\section{INFLUENCE OF NOISE ON THE IF ESTIMATION}

Consider a discrete signal $s(n)=r e^{j f(n)}$ corrupted by a Gaussian white noise $v(n)$. Without loss of generality, the discrete complex-lag distribution $N=4$, can be written as [7]:

$$
\begin{aligned}
& G C D_{N=4}(n, w)=\stackrel{N_{s} / 2}{\mathrm{a}} w(m)(s(n+m)+n(n+m)) \\
& m=-N_{s} / 2
\end{aligned}
$$

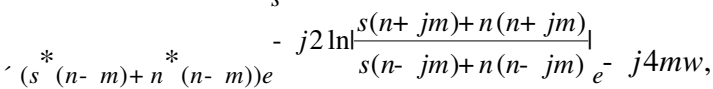

where $m$ is a discrete lag coordinate, $N_{s}$ is the number of signal samples, while $w(m)$ is a window. If the small noise is assumed, the following approximation can be used:

$$
\begin{aligned}
& \ln |s(n+j m)+n(n+j m)|=\ln |s(n+j m)|+\mathrm{D} q_{n}(n, m), \\
& \mathrm{D} q_{n}=\operatorname{Re}\{n(n+j m) / s(n+j m)\},
\end{aligned}
$$

where $\mathrm{D} q_{n}$ is a phase deviation of the terms with complex-lag argument. The values of signal with complex-lag argument are calculated using the analytical extension as follows [7]:

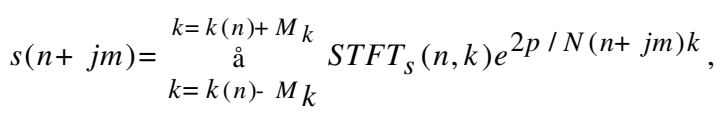

where STFT denotes the short time Fourier transform, while the width of the signal component in the TF plane is $2 M_{k}+1$. The same relation holds for the noise $n(n+j m)$. The bias and the variance of the IF estimate for the complex-lag distribution of order $N=4$ are derived as [7]: 


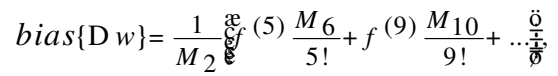

$$
\begin{aligned}
& \operatorname{var}\{\mathrm{D} w\}: \frac{s_{n}^{2} N_{w}^{3}}{24 r^{2} M_{2}^{2}}\left(\frac{1}{4}+\frac{3}{8 p N_{w}} e^{2 p N_{w} M_{k} / N_{s}}\right)
\end{aligned}
$$

where the rectangular window of width $N_{w}$ is used. Although the $\operatorname{GCD}_{N=4}(n, w)$ is of higher order $N$ comparing to the Wigner distribution, for the values: $N_{s}=128, N_{w}=16, M_{k}=8$, the variance in $G C D_{N=4}$ is of the same order as the variance in the Wigner distribution, while the bias is significantly lower.

In the sequel we provide analysis of noise influence in the case of $L$-complex-lag distribution $(N=4)$. For a noisy signal $x(n)$, under the low-noise assumption, we can use the following model with additive noise:

$$
x^{L}(n)=(s(n)+n(n))^{L} @ s^{L}(n)+L s^{L-1}(n) n(n)=s^{L}(n)+x(n),
$$

where the autocorrelation function of $x(n)$ is defined as: $R_{x x}(n)=r^{2 L-2} s_{n}^{2} L^{2} d(n)$, while $\sigma_{v}$ is the variance of noise $v(n)$. Therefore, the discrete form of $L$-complex-lag distribution for noisy signal can be defined as:

$$
\begin{aligned}
& C T D_{N=4}^{L}={\underset{\mathrm{a}}{\mathrm{a}}}_{m=-N_{S} / 2}^{N_{S} / 2} w(m)\left(s^{L}\left(n+\frac{m}{L}\right)+x\left(n+\frac{m}{L}\right)\right)\left(\left(s^{*} L\left(n-\frac{m}{L}\right)+x *\left(n-\frac{m}{L}\right)\right)\right. \\
& e^{-j 2 L q(n, m)} e^{-j 4 m w} \text {, }
\end{aligned}
$$

where: $q(n, m)=\ln \left|s\left(n+j \frac{m}{L}\right)+n\left(n+j \frac{m}{L}\right)\right|$. The bias and variance of the IF estimate are obtained as follows:

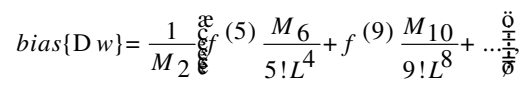

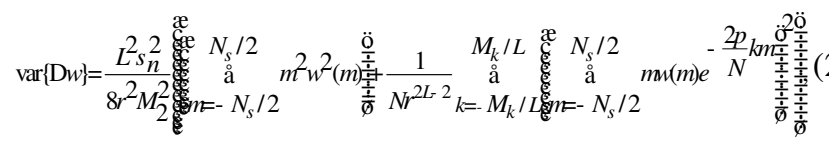

In the case of rectangular window $w(m)$ of length $N_{w}$, we have:

$$
\operatorname{var}\{\mathrm{D} w\}: \frac{L^{2} s_{n}^{2} N_{w}^{3}}{24 r^{2} M_{2}^{2}}\left(\frac{1}{4}+\frac{3}{8 p r^{2 L-2} N_{w}} e^{2 p N_{w} M_{k} / N_{s} / L}\right)
$$

If the second term within the brackets is small enough, the variance in the $L$-complex-lag distribution $(N=4)$, is of the same order as the variance in the $L$-Wigner distribution $(N=2)$.

For example, $3 \exp \left(2 p N_{w^{M}} M_{k} / N_{s} / L\right) / 8 p r^{2 L-2} N_{w}=0.17$ stands for $N_{s}=128, N_{w}=16, M_{k}=8, r=1, L=2$. Therefore, the variance of the IF estimation based on the $L$-complex-lag distribution $(N=4)$ is preserved on the same level as in the case of $L$ Wigner distribution, while the bias is significantly reduced.

\section{EXAMPLE}

Consider the highly non-stationary signal in the form: $x(t)=\exp (j \cdot(2 \cos (2 \cdot \pi \cdot t)+1 / 2 \cos (6 \cdot \pi \cdot t)+1 / 2 \cdot \cos (4 \cdot \pi \cdot t)))+v(t)$, where $v(t)$ is complex white Gaussian noise $(\mathrm{SNR}=30 \mathrm{~dB})$. The time interval $t \in[-1,1]$, with $\Delta t=2 / 128$ is used. The results obtained for Wigner distribution, L-Wigner distribution, $G C D_{N=4}, G C D_{N=4}^{L=2}, G C D_{N=6}$ are given in Fig 3. Note that for the considered signal, the $G C D_{N=4}^{L=2}$ improves the concentration of $G C D_{N=4}$ by reducing the inner-interferences, while it is less sensitive to noise comparing to $G C D_{N=6}$. In this case the Wigner distribution and its L form are useless for the IF estimation.

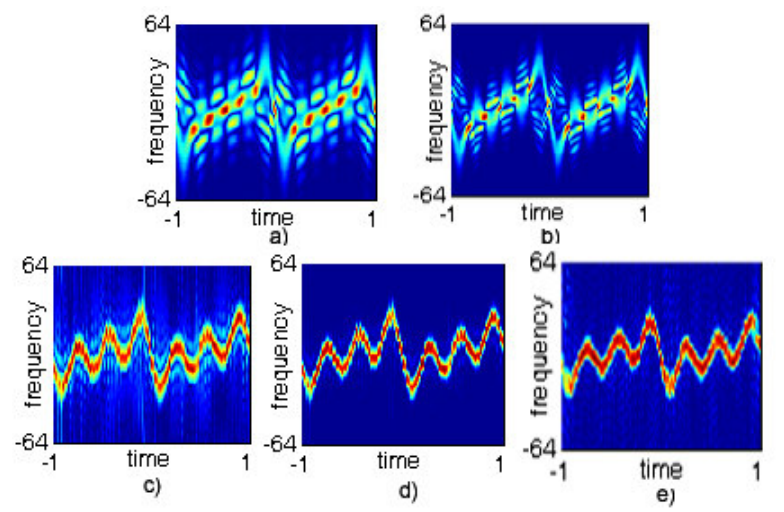

Fig 3. a) Wigner distribution, b) $L$ Wigner distribution, c) $G C D_{N=4}$,d) $G C D_{N=4}^{L=2}$, d) $G C D_{N=6}$

\section{CONCLUSION}

The analysis of TF distribution concentration based on the concept of Cauchy's integral formula discretization is provided. The two aspects of concentration improvement are considered: the number of circle points used in the discretization and the number of integrations. It has been shown that the suitable choice of the circle points enables removing of certain undesirable derivatives in the spread factor, providing an accurate IF estimation. As a result of this analysis, the general forms of complex-lag TF distributions are defined, as well as their $\mathrm{L}$ forms. Some of the well-known TF distributions are obtained as their particular cases.

\section{REFERENCES}

[1] B. Boashash, "Time-Frequency Analysis and Processing," Elsevier, 2003, Amsterdam

[2] B. Barkat, and B. Boashash, "Design of higher-order polynomial Wigner-Ville distributions", IEEE Trans. Signal Process., vol. 47, No. 9, pp. 2608-2611, Sept. 1999.

[3] R. L. Murray, A. Papandreou-Suppappola, G. F. Boudreaux-Bartels, "New higher order spectra and time-frequency representations for dispersive signal analysis," IEEE Int. Conf. on Acous., Speech and Signal Proc., vol. 4, pp. 2305-2308, May 1998.

[4] G. Viswanath and T. V. Sreenivas, "IF estimation using higher order TFRs," Signal Processing, vol.82, No.2, pp.127-132, Feb. 2002.

[5] M. Morelande, B. Senadji, and B. Boashash, "Complex-lag polynomial Wigner-Ville distribution," Proc. of IEEE Speech and Image Technologies for Computing and Telecom., vol.1, pp. 43-46, Dec. 1997.

[6] LJ. Stanković, "Multitime Definition of the Wigner Higher Order Distribution: L-Wigner Distribution," IEEE Signal Processing Letters, vol.1, No.7, July 1994.

[7] LJ. Stanković, "Time-Frequency distributions with complex argument," IEEE Trans. Signal Processing, vol. 50, No..3, pp.475-486, Mar. 2002.

[8] C. Cornu, S. Stanković, C. Ioana, A. Quinquis, LJ. Stanković, "Generalized Representation Derivatives for Regular Signals," IEEE Trans. on Signal Processing, vol..55, No.10, pp.4831-4838, Oct.2007.

[9] S. Stanković, N. Žarić, I. Orović, C. Ioana, "General form of the timefrequency distribution with complex-lag argument," Electronics Letters, vol. 44, No. 11, pp. 699-701, May 2008. 